\title{
Large surveys and the Virtual Observatory
}

\section{Konrad Kuijken}

Leiden Observatory, Leiden University, PO Box 9513, NL-2300RA Leiden, the Netherlands email: kuijken@strw.leidenuniv.nl

Increasingly, large surveys of the sky in multiple wavelength bands are becoming an important part of how astronomy is being done. These surveys produce huge homogeneous datasets, and as such are prime material for dissemination and analysis through the VO. As an example, the KIDS survey (see Fig. 1), which will map 1500 square degrees of sky in ugriZYJHK using ESO's VST and VISTA telescopes, and which is expected to start in 2007, will generate some $15 \mathrm{~TB}$ of pixel data, as well as several TB of derived source parameters. More than 100 TB of raw data will have to be processed.

Analysis of such massive datasets requires that the end users are empowered to interact with their data flexibly. Given the huge data volumes, it will often be much more efficient to turn calculations into complex queries on the database, particularly so if other data sets (X-ray or radio maps, or earlier epochs from older surveys such as SDSS, for example) are to be incorporated.

Surveys help the VO by populating it with well-qualified data, while the VO helps the community to extract the science from this vast resource.

A critical application is to measure accurate colours for extended objects (galaxies), in order to derive photometric redshifts. Since different wavebands will typically be observed in different seeing conditions, or even at different telescopes with different pixel scales, this is not trivial. The development of techniques that correct the photometric catalogues for different image quality is vital if colours can be measured efficiently; the traditional technique of convolving pairs of images to a common seeing is simply too laborious when half a dozen different surveys are considered. Details on KIDS survey can be found at <http://www.strw.leidenuniv.nl/ kuijken/KIDS/>.
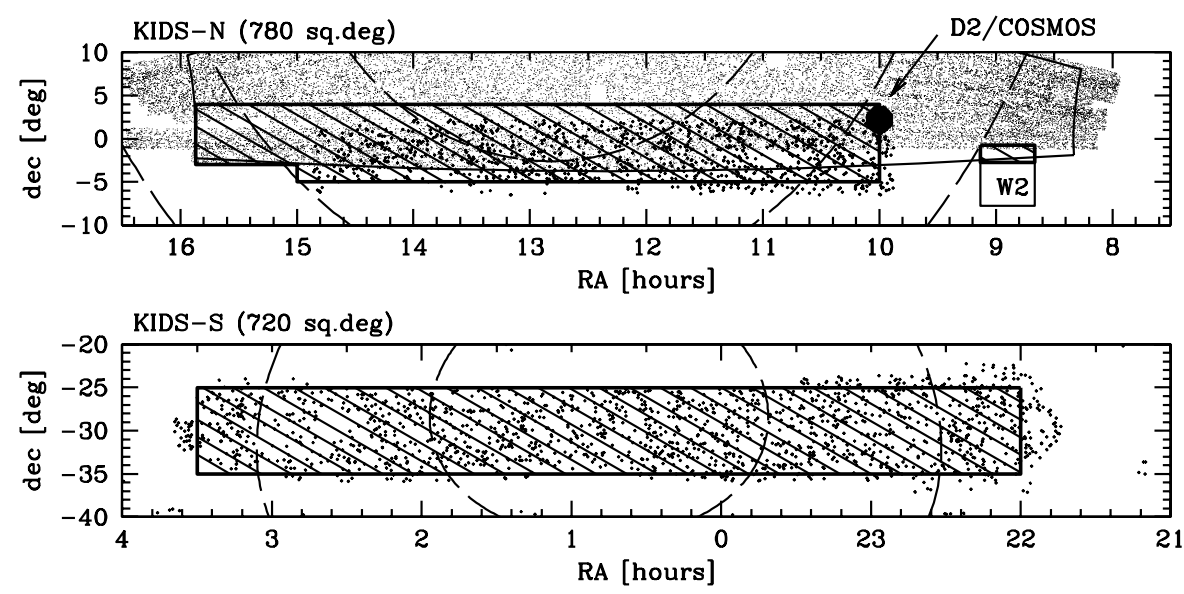

Figure 1. Layout of the KIDS survey fields, with SDSS (dots) and 2dF (crosses). 